\title{
Closed/Open-Cell Photoacoustic Imaging for Spectroscopic Measurements
}

\author{
Tsutomu Hoshimiya \\ Department of Electronic Engineering, Tohoku Gakuin University, Tagajo, Japan \\ Email: tpth@mail.tohoku-gakuin.ac.jp
}

Received 11 February 2016; accepted 8 August 2016; published 15 August 2016

\begin{abstract}
Photoacoustic imaging using a closed photoacoustic cell and an open photoacoustic cell with gasmicrophone detection scheme was described. R/G/B LED light sources were used for the closed photoacoustic (PA) cell configuration. The colored specimen enclosed in a PA cell was imaged with R/G/B color light sources, and an image restored from the inverted PA images was compared with the original image. For open cell configuration, an open PA cell using a spheroidal acoustic resonator was applied to measure the amount of large-sized colored specimens. A calibration curve for a food red dye was obtained that apparently showed the ability of the present scheme to measure as a spectroscopic measurement tool.
\end{abstract}

\section{Keywords}

Photoacoustic, Imaging, Spectroscopic, Calibration Curve, Color Restoration

\section{Introduction}

In the photoacoustic microscopy (PAM) or photoacoustic (PA) imaging, gas-microphone and piezo-electric detections have been used for the spectroscopic imaging or nondestructive evaluation (NDE) of specimens. In a gas-microphone detection, specimens must be contained in an air-sealed PA cell. On the other hand, a piezoelectric transducer for the PA imaging has to be attached to the specimen. In both cases, the limitation of specimen size should occur.

In the spectroscopic analysis with PA imaging, PA imaging apparatuses with monochromatic light sources have been used and were applied to measure a paper chromatography [1], dry chemical analysis [2] and pollen measurement [3] up to now. However, these applications to imaging of microscale spectroscopic objects with PAM prefers a multiple-wavelength optical source rather than a monochromatic one and an ability to analyze large-size specimens is also required.

In the present study, we tried to open the way to establish a spectroscopic measuring apparatus with both i) wide spectral range and high spectral resolution and ii) high spatial resolution with large-size scanning region by two different approaches. One approach is to use multiple wavelengths light sources which can detect specimen color with the ability to measure its amount for objective i), and the other approach is to develop an effective coupling between PA detector (microphone) and the specimen located outside the PA cell in order to measure a large-sized spectroscopic specimen for objective ii). 
In this paper, two types of apparatus were reported. One is a PA imaging apparatus with a multiple fibercoupled LED light sources combined with the incidentmicroscope for a closed cell configuration [4]. The other is aPA cell with a spheroidal acoustic resonator [5] working as an acoustic coupler from sound generated at the specimen to a detector (high-sensitive condenser microphone) for open resonant cell configuration [6].

The results with a former apparatus were shown with an original color restoration technique. The results with the latter apparatus were summarized as a calibration curve for powdered dye amount measurements.

\section{Experimental Apparatus, Specimens and Procedures}

\subsection{Closed Cell Configuration}

A basic experimental setup for multiple-wavelength LED photoacoustic microscope (PAM) was shown in Figure 1. As optical sources, high-power fiber-coupled LEDs (Thorlabs) were used. A 4-channel modulator (Thorlabs, DC4104) was used to modulate them. The wavelengths and powers of red, orange, green and blue colors LEDs were $660 \mathrm{~nm} / 10.8 \mathrm{~mW}, 617 \mathrm{~nm} / 7.62 \mathrm{~mW}, 505 \mathrm{~nm} / 9.82 \mathrm{~mW}$, and $455 \mathrm{~nm} / 16.4 \mathrm{~mW}$, respectively. Plastic optical fibers with a $1.0 \mathrm{~mm}$ diameter and $120 \mathrm{~mm}$ length (Mitsubishi Rayon, SK-40) were used to guide light beams to incident a microscope objective lens $(\times 20)$. Between combined fibers and a microscope objective, a 10 mm diameter ball-lens was inserted to improve coupling efficiency. Modulation frequency was $90 \mathrm{~Hz}$. A high-sensitive condenser microphone (Brewer \& Kaejer 4166) and a lock-in amplifier LI-5610B (NF Circuit block) were used for detection. Scanning area was $30 \mathrm{~mm} \times 30 \mathrm{~mm}$ and performed with a linear-motor stages (Chuo-seiki, ALD-105-H1L). The image resolution was $60 \times 60$ pixels.

\subsection{Open Resonant Cell Configuration}

The basic experimental setup was shown in Figure 2. A PA cell with a prolate spheroidal acoustic cavity with axial length $(2 \mathrm{a}=81 \mathrm{~mm})$ and diameter $(2 \mathrm{~b}=27 \mathrm{~mm})$ was used for an acoustic resonator. The fundamental spheroidal resonant frequency of the cavity was calculated to be $2860 \mathrm{~Hz}$ For a PA cell, one side of spheroid at the focus was cut for an opening attached to a specimen under study. At the other focus, a high-sensitive condenser microphone (Bruel and Kaejer 4166) was attached. And the other hole was drilled as a window for the laser incidence at the side wall of the resonator. For an optical source, a Nd-YAG diode-pumped solid-state laser (DPSSL) with a SHG wavelength of 532nm (maximum power $500 \mathrm{~mW}$ ) was used. Laser beam with the power of $125 \mathrm{~mW}$ was pulse modulated with a function generator (NF Circuit Block, DF1906). A PA signal was fed into a lock-in amplifier (NF-Circuit Block, LI-5610B) and detected phase-sensitively. In the case of imaging, a specimen was scanned with a XY-linear motor stages.

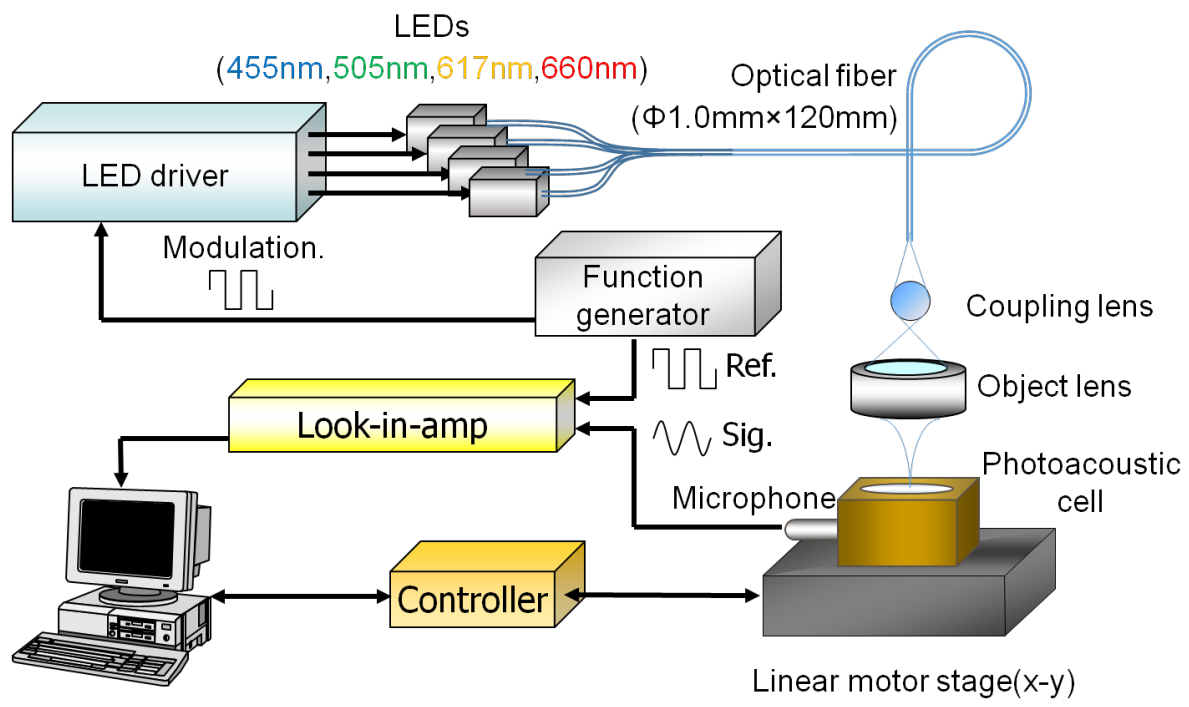

Figure 1. Basic experimental setup for closed cell configuration (Ref. [4]). 


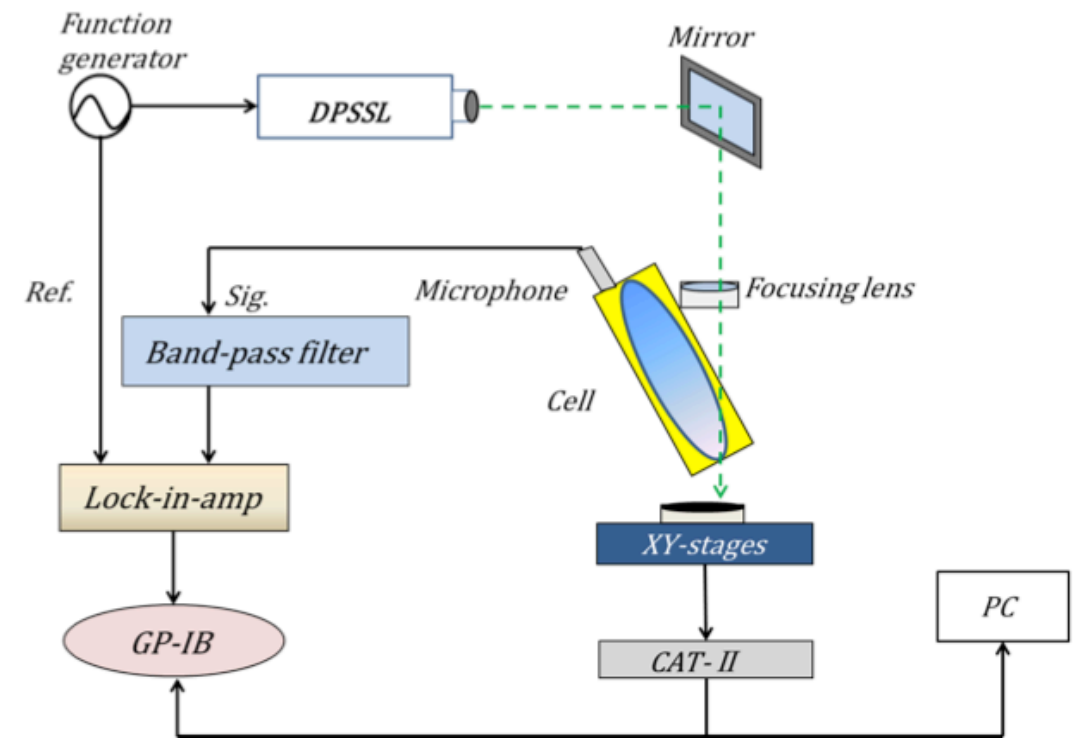

Figure 2. Basic experimental setup for open cell configuration.

\subsection{Specimen for Closed Cell with R/G/B Color Excitation}

For a colored sample, an image of the Mickey Mouse (ㄷ Walt Disney) printed on a transparent polyethylene terephthalate sheet was used. A $15 \mathrm{~mm} \times 15 \mathrm{~mm}$ square was also drawn around it. For every wavelengths, PA images were recorded, respectively. The obtained PA images for every red, orange, green and blue excitation were firstly shown in 256-grade gray-color.

\subsection{Specimen for Open Resonator Cell}

For spectroscopic measurement applications, the large dynamic-range spectroscopic detection ability of the PA imaging, as demonstrated by pollen detection [1] and dry chemicals [2], is important. To increase this advantage, the author applied the spheroidal acoustic resonator to the PA cell with a large aperture for coupling of the acoustic signal generated at a specimen located near outside focus of the spheroid with a microphone located at the inside focus of the spheroid [3]. A red-colored food dye powder was used as a specimen, which absorbs green $(532 \mathrm{~nm})$ laser light well. PA signals were integrated over the specimen surface with changing the amount of the dye so that calibration curve was obtained.

\section{Experimental Results}

The original color restoration procedure was as follows: The gray-images obtained with R/G/B LEDs were colored with their complementary colors (cyan/ magenta/ yellow).

The transmittance spectra of the seven colors (red, green, blue, cyan, mazenta, yellow, and black)were shown with their B/G/R 0-255 level digital color component. After making a gray-scale PA image of the specimen, PA image corresponds to absorbance of the sample at the irradiation wavelength. So the inversion of the black/white tone of the PA image corresponds to the transmittance (reflectivity) of the specimen at the irradiation wavelength.

Based on the principle described above, the images inverted from the obtained R/G/B images was colored with colors corresponding to the irradiation wavelngths, and then combined. The color image reconstruction procedure for sample \#2 (Mickey Mouse) was shown in Figure 3. The obtained restored image was compared with the original image shown in Figure 4. The restored image was blurred due to the imcomplete focusing with LED source, however color restoration is remarkable.

\subsection{R/G/B Color PA Imaging and the Color-Image Restoration (Closed Cell Configuration)}

Show as Figure 3. 

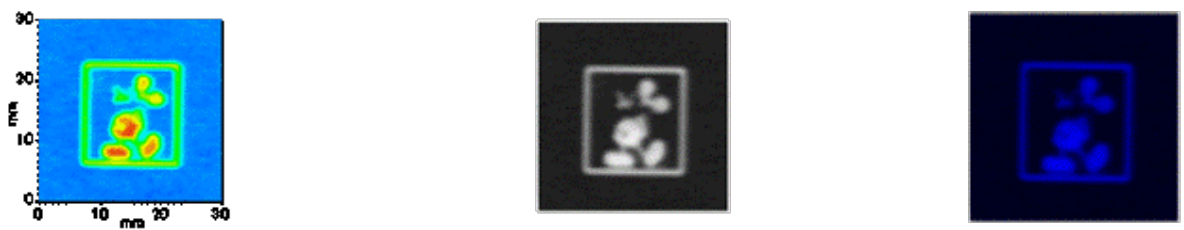

PA image (455nm)
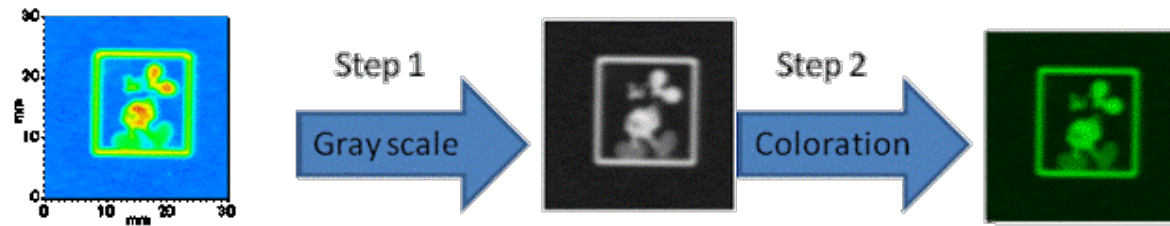

PA image (505 $\mathrm{nm})$
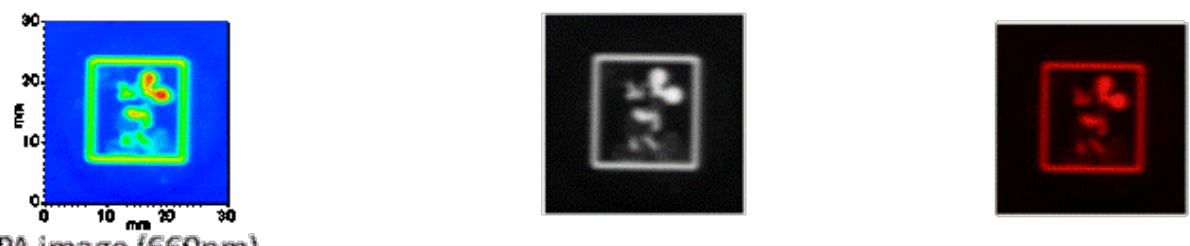

PA image (660 nm)

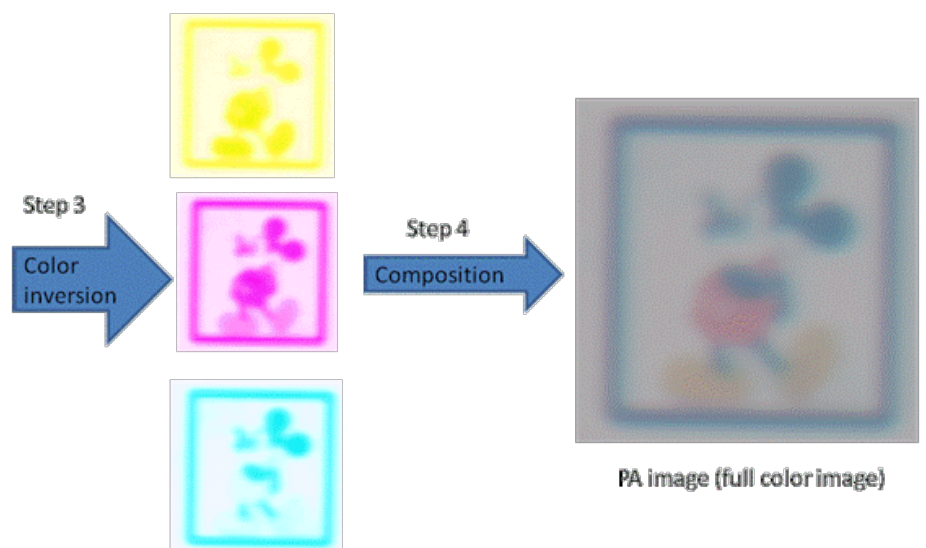

Figure 3. Color image restoration procedure.

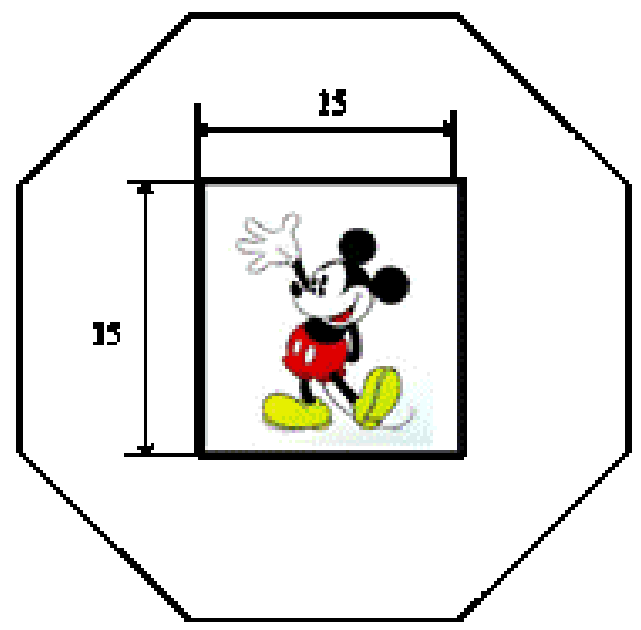

Figure 4. Original image. 


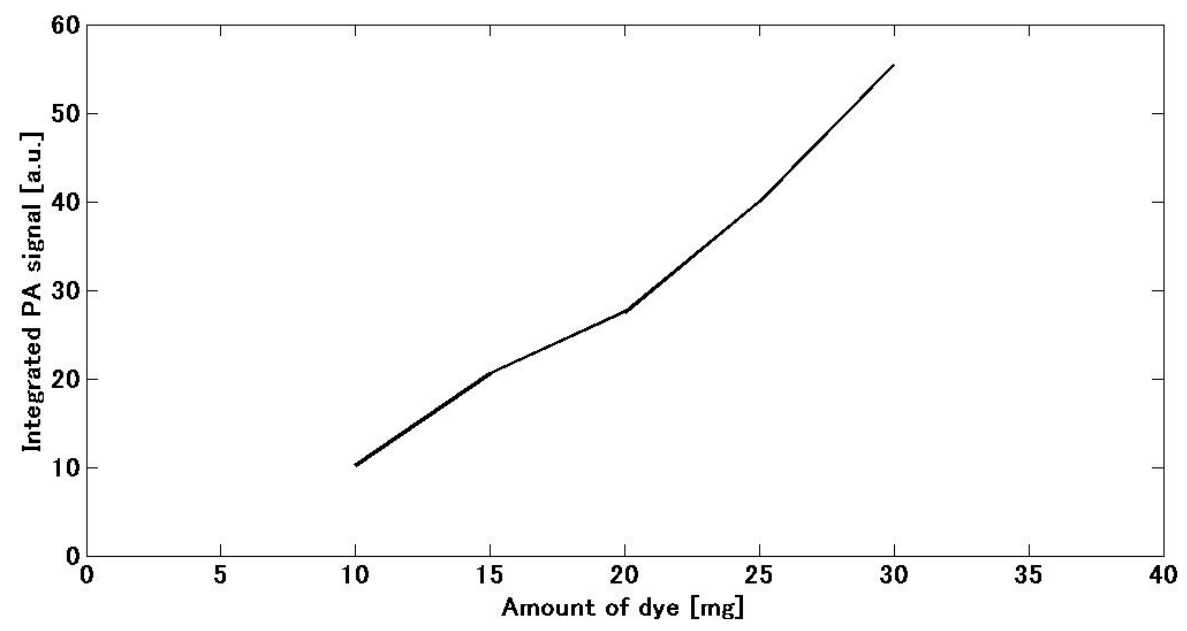

Figure 5. Caliblation curve for red food dye.

\subsection{Red Color Dye Spectroscopic Measurement (Open Cell Configuration)}

Red food dye powders with the amount ranging 10 - $30 \mathrm{mg}$ were used for the specimens. For each specimen, generated PA signal was integrated over the specimen surface set on an aluminum foil irradiated by the focused laser beam with the power of $22 \mathrm{~mW}$. The PA signal integrated over the specimen surface (PA signal recorded as a csv data were summed along each columns and then summed along all rows with Excel) was plotted with changing the amount of the dye. The resultant calibration curve was shown in Figure 5.

This scheme will help to measure a large-sized substances such as paper and thin-layer chromatographs used in analytic chemistry as shown in the previous publications [4].

\section{Discussions and Conclusion}

In this paper, two types of gas microphone detection apparatus were used to apply the spectral analysis. Firstly the spectral-analyzing ability of the present PA imaging scheme was shown by full-color restoration in a closed cell configuration. Secondly the ability of the spectral analysis of substances using the PA imaging in the open cell configuration using a spheroidal acoustic resonator was demonstrated by dye powder amount measurement.

\section{References}

[1] Hoshimiya, T. (1990) A Rapid-Scan and High-fidelity Dual-Mode Photoacoustic Microscope and Its Applications to Paper Chromatography. In: Murphy, J.C., Ed., Photoacoustic and Photothermal Phenomena II, Springer Verlag Optical Science Series. http://dx.doi.org/10.1007/978-3-540-46972-8_137

[2] Hoshimiya, T. (1992) Evaluation of Dry Chemicals by Photoacoustic Imaging. Japanese Journal of Applied Physics, 31, 3474-3476. http://dx.doi.org/10.1143/JJAP.31.3474

[3] Miyamoto, K. and Hoshimiya, T. (2006) Measurement of the Amount and Number of Pollen Particles of Cryptomeria japonica (Taxodiaceae) by Imaging with a Photoacoustic Microscope. IEEE Transactions on Ultrasonics, Ferroelectrics, and Frequency Control, 53, 586-591.

[4] Adachi, Y. and Hoshimiya, T. (2013) Photoacoustic Imaging with Multiple-Wavelength LEDs. Jpn. J. Appl. Phys., 52, 07 HB06.

[5] Chang, C.T.M. (1970) Natural Resonant Frequency of a Prolate Acoustical Resonator. Journal of Acoustical Society of America, 49, 611-614. http://dx.doi.org/10.1121/1.1912399

[6] Hoshimiya, T., Hoshimiya, J. and Hayasaka, Y. (2012) Resonant Photoacoustic Detection and Imaging with an Ellipsoidal Acoustic Resonator. Proceedings of IEEE Ultrasonics Symposium, Dresden, P5F-4. 


\section{Submit or recommend next manuscript to SCIRP and we will provide best service for you:}

Accepting pre-submission inquiries through Email, Facebook, LinkedIn, Twitter, etc.

A wide selection of journals (inclusive of 9 subjects, more than 200 journals)

Providing 24-hour high-quality service

User-friendly online submission system

Fair and swift peer-review system

Efficient typesetting and proofreading procedure

Display of the result of downloads and visits, as well as the number of cited articles

Maximum dissemination of your research work

Submit your manuscript at: http://papersubmission.scirp.org/ 